Original Research Paper

\title{
Space Debris Removal Mechanism Using CubeSat with Gun Shot Facilities
}

\author{
${ }^{1}$ Shankar Bhattarai and ${ }^{2}$ Jie-Rou Shang

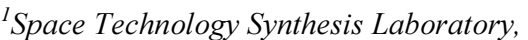 \\ Department of Aerospace Engineering, Chosun University, Gwangju, Republic of Korea \\ ${ }^{2}$ Institute of Astronomy, National Tsing Hua University, Taiwan
}

Article history

Received: 16-04-2018

Revised: 24-08-2018

Accepted: 15-11-2018

Corresponding Author:

Shankar Bhattarai

Space Technology Synthesis

Laboratory, Department of

Aerospace Engineering,

Chosun University, Gwangju,

Republic of Korea

Email: oshobhattarai2@gmail.com

\begin{abstract}
Space derbies is a very perilous problem and most of the proposed methods for space debris removal are not successfully serviceable for all sized space junk. Keeping in view the limitation of the existing methods of debris removal techniques an active approach is needed and in this regard required to be instigated purposefully. In more than half a century of space activities, by using above 4800 launches have placed around 6000 satellites into orbit, of which less than a thousand are still operating. In this research, a new concept to remove small space junks of Low Earth Orbit (LEO) is proposed which functions by using CubeSat with Gunshot equipment. The target of this concept is to confiscate the small-sized space junk of ranges 0.2 to 1 inches diameter and which will be identified by collaborating with FGAN radar. CubeSat is loaded five bullets of $0.1 \mathrm{~kg}$ with strong thruster gunshot technology and after the first shot, reaction force will help to change the orbit of the satellite to find out the second space debris. This research intended to comprehend and helps to get assess of small-sized space debris removal activity in terms of the simple business model.
\end{abstract}

Keywords: Space Debris, CubeSat, Low Earth Orbit, Gunshot Mechanism

\section{Introduction}

Since the beginning of the space activities, debris as small as dots of point and as large as whole satellite and parts of rockets have been accumulating in space. It is assumed that millions of pieces of junk are whizzing around our planet earth. The probability of Spacecraft being hit at any piece of debris is currently low due to space is very big and anxieties are growing because of promptly growing space activities. Debris poses a growing threat to satellites and could prevent the use of valuable orbits in the future (David, 2007). Many pieces of debris are too small to monitor but too large to shield satellites against. In a more than half-century of our space age, space junk is now become perilous in Low Earth Orbit (LEO), defined as the region of space around Earth with an altitude of $160-2000 \mathrm{~km}$. The density of objects in LEO will most likely increase due to new launches, on-orbit explosions and object collisions.

More than 12,000 orbiting items in total are regularly tracked by the US Space Surveillance Network and maintained in their catalog, which covers objects larger than approximately $5 \mathrm{~cm}$ to $10 \mathrm{~cm}$ in Low Earth Orbit
(LEO) and $30 \mathrm{~cm}$ to $1 \mathrm{~m}$ at Geostationary altitudes (GEO) (Johnson, 2009). Satellites are used in many critical military and civilian applications. There are over 900 active satellites in orbit around the Earth, of which about half are operated commercially and half by governments and the military. Approximately two-thirds of active satellites are used for communications. The rest are split roughly equally between navigation, military surveillance, Earth observation and remote sensing, astrophysics and space physics and Earth science and meteorology (Wheeler, 2007). Satellites orbit the Earth at a range of altitudes and orientations depending on their function. Space debris generally refers to manmade material in orbit that no longer serves a useful purpose. Because of the high speeds of objects in orbit $(7.5 \mathrm{~km} / \mathrm{s}$ is typical in low earth orbit), even small pieces of debris can be very damaging in a collision (Sannigrahi, 2017). There are several types of debris; defunct spacecraft: such as satellites that have ended their useful life, commercial satellites have an average lifespan of only around 15 years, spent rocket bodies used to launch satellites into orbit; objects released 
during missions, such as waste vented from the space shuttle and small fragments caused by collisions, explosions or deterioration of active satellites or larger pieces of debris (Kessler and Cour-Palais, 1978). Debris is placed in one of three categories according to its size and potential impact. Debris between $1 \mathrm{~cm}$ and $10 \mathrm{~cm}$ is known as the fatal population because it is large enough to destroy a satellite but too small to be tracked from earth. Therefore remediation effects should not be directed exclusively towards the removal of large junk, but must also consider the removal of small sized debris.

\section{Recent Debris Incidents}

In 2016 ESA's satellite Sentinel 1A, which was launched in 2014 encountered the small debris accident on its solar panels and affect power supply of Satellite. Other incidents of debris creation have involved the deliberate destruction of a satellite by missiles launched from Earth. In January 2007, China destroyed one of its defunct weather satellites orbiting at about $900 \mathrm{~km}$. This created around 2700 new pieces of tracked debris and NASA estimates more than 150,000 pieces of debris larger than $1 \mathrm{~cm}$ were created (Bonnal et al., 2013; Ansdell, 2010). When the US destroyed one of its satellites in February 2008, few lasting pieces of debris were created as the satellite was in a lower orbit, from which most debris rapidly re-entered the atmosphere. In February 2009, the first collision between two satellites occurred $800 \mathrm{~km}$ above Northern Siberia. One was an active US communications satellite, while the other was a defunct Russian satellite. They collided at a speed of over $40,000 \mathrm{~km} / \mathrm{h}$, causing the complete break-up of both satellites. The event created around 1400 cataloged debris objects (NPDPO, 2007).

There have been several incidents producing the high number of debris. Two of them have been especially severe: (i) A 2007 Chinese anti-satellite missile test producing more than 1200 catalogued pieces of debris and an estimated 35,000 pieces of a size of $1 \mathrm{~cm}$ and larger, resulting in the most severe orbital debris cloud in history (NPDPO, 2009) (ii) the collision of the Iridium33 and Kosmos-2251 satellites in 2009, which was the first accidental hypervelocity collision of two intact spacecraft (Nishida and Yoshikawa, 2003). Fig. 1 shows the growth rate of debris in space environment.

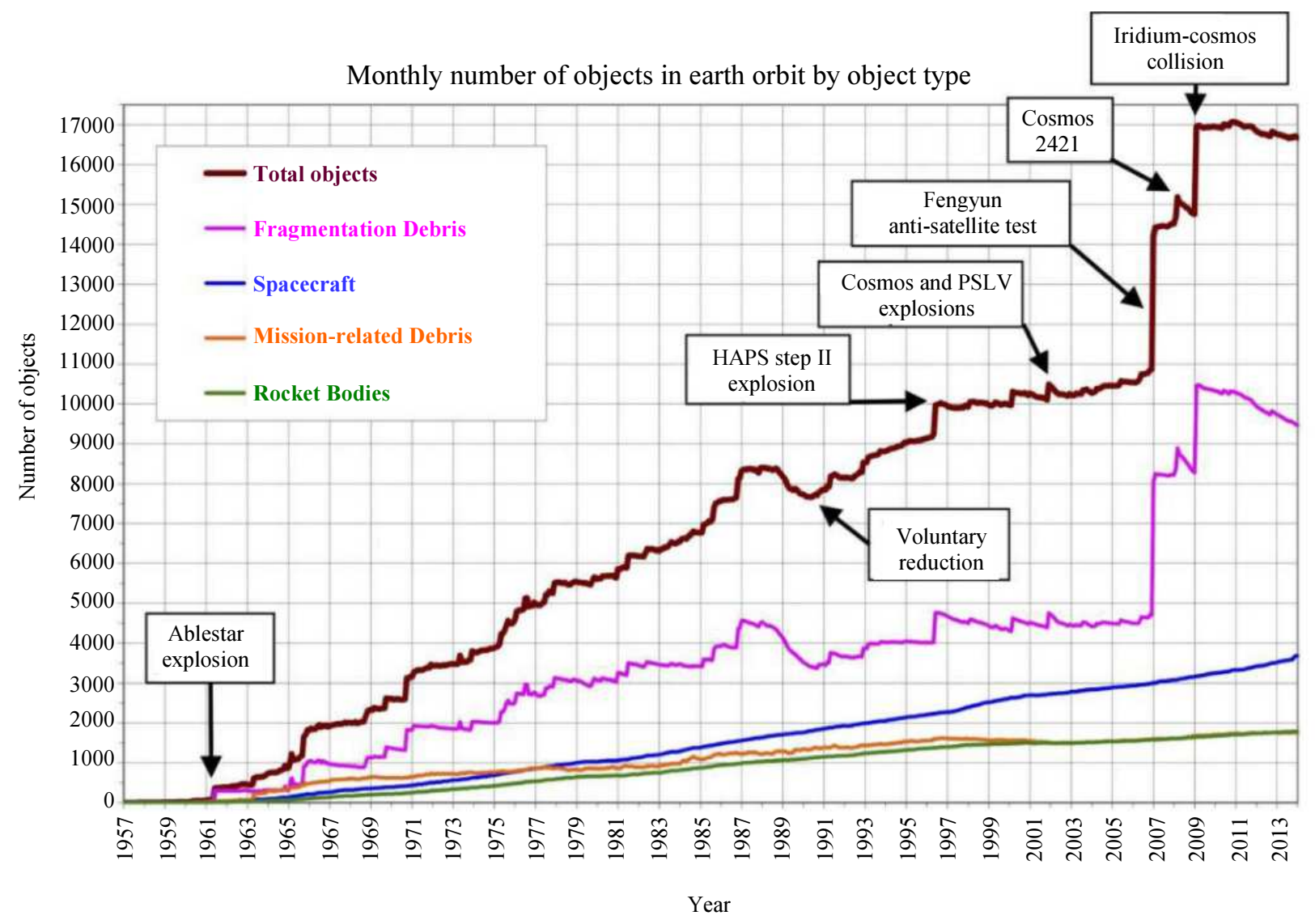

Fig. 1:The timeline of the growth in the number of objects being tracked in orbit. Due to limited tracking capabilities (limited to larger than $10 \mathrm{~cm}$ ), the actual number of objects in orbit is estimated to be 2 to 3 times the numbers in the figure (Nishida and Yoshikawa, 2003) 
Currently, collisions are the smallest contributor to fragments of debris. However, as the number of debris objects increases, collisions become more likely, thus creating yet more debris (COPUOS, 2007). The cascade process is known as the Kessler syndrome and space experts argue that without mitigation it could make it impossible to operate satellites safely in the future. Several techniques have been proposed for the removal of debris, some are by attaching a propulsion device to a debris object to push it out of orbit, using a robotic grappling device on another spacecraft to pull an object to a new orbit or to cause it to re-enter the atmosphere grievously or using a momentum exchange tether; which acts like a swing to pull an object out of orbit. And by using an electrodynamic tether, which causes a drag on the satellite due to the magnetic field of the Earth besides that slowing objects using high-powered lasers fired from Earth, so that they move out of orbit (Bastida and Krag, 2011). But all of these techniques are focused on big sized debris removable process and not equally suitable for small sized. NASA and ESA research showed that the environment can be stabilized; when on the order of 10 objects are removed from LEO per year with a removal sequence oriented towards the target mass. The main problem with accessing existing pieces of debris is the fuel expenditure needed to reach more than one piece of debris per launch. However, CubeSat with Gunshot technology is going to be very effective because of its multiple derbies removable mechanism and we believe that there is potential in the future for a commercial removal service.

\section{Gunshot Technology for Deorbiting the Small Debris}

In this research intend to use Gunshot technology in small satellites, which is design as shown in Fig. 3. It is totally a new debris removable concept and planning to fixed small gun technology with five bullets in a Cube Satellites. The main goal of debris removable with the help of Gunshot is to demonstrate the feasibility of removing small sized junk. Such a solution is much safer than a contract-based mechanism and applicable to the quite small class of different shaped space objects. Consequently, it allows the large distance between the target and the Satellite, lowering the collision occurrence chance. The vigorous design of the gun-bullet based solution attention must be paid in position, weight and sized of the debris, which leads to the bullet to correctly hit to the target. To this end, modeling, numerical simulation and experimental testing are fundamental works. To get the goal, firstly a simulation of the thrust release from the bullet and the energy required to lower down the orbit of debris is needed. Secondly, a test must be implemented to accomplish experimental tests to deeply understand the dynamics and correctly refine the mechanism design. The technical generalities are firstly presented; the mechanism and crucial parts design dynamics, numerical simulations and optimization process will be discussed.

\section{The Mechanism of Monitoring Debris}

Debris is monitored from Earth using radar (for LEO) and telescopes. This allows larger and smaller pieces of debris to be tracked and cataloged. The largest catalog is maintained by the US military, using its worldwide Space Surveillance Network (SSN), which includes the Fylingdales radar base in the UK. It contains around 19,000 objects (Liou, 2009). However, only relatively crude information about the orbital paths is made available for wider use; and no information at all on around $25 \%$ of the objects for security and other reasons (Benvenuto and Carta, 2013). While it is desirable to extend tracking to smaller objects, this would lead to a huge increase in the number of objects in the catalog. For example, the US Space Fence radar system, scheduled to come online in 2015 , is expected to add 100,000 or more new objects in the $1-10 \mathrm{~cm}$ range to the SSN catalogue (Nielsen et al., 2012). Modifying the catalogue to accommodate these data will be complex and expensive.

The properties of debris that is too small to be observed directly can be inferred by examining its impacts on satellites such as the International Space Station (ISS) and the Hubble telescope, which is visited regularly by Space Shuttle missions (Golebiowski et al., 2016). When the debris is trappable from the satellite, first we have to identify it; collaborating with FGAN radar. The FGAN radar is sensitive enough to detect 2 $\mathrm{cm}$ sized objects at a distance of $1000 \mathrm{~km}$ (Mehrholz et al., 2002). After targeting, firing the bullet to the targeted debris as shown in Fig. 2.

Additional requirements include that the Gun shall fully mark the debris right before the target is reached, to start the consistently designing and finally implementing the system, a numerical analysis-based on a dedicated simulator run first. As reported in the followings, numerical simulations provided the relations between the bullets and Cube Satellite masses, the bullets initial velocity vectors to size the gun and bullet shooting mechanism.

\section{CubeSat Designing and Numerical Analysis}

The main aim of the implemented similar stays in studying the Gun dynamics from the shot, through the debris removable, to tune some configuration key elements in order to achieve the expected dynamics. The most challenging modeling aspect relates to the Gun 
efficiency, which is very effective in dealing with flexible structure modeling, taking the advantages of shape functions to satisfy the boundary conditions and to decouple in space from time variation aspects. However, we have developed the general applied to design with quite simple configurations that include few flexible elements. And with the coexistence of many flexible structures, the problem structuring requires an approach based on simpler methods, always capable of describing the phenomenon and easier to handle. The platform, shown in Fig. 3 is based on four side panels and $3 \mathrm{U}$ Payload System. The side and payload panels will be made from aluminum honeycomb or machined aluminum. This platform subsystem will includes MEMS thruster, Antenna, Solar Panel, ADCS Models, OBC, EPS, Gun, Bullet and Debris Detector.

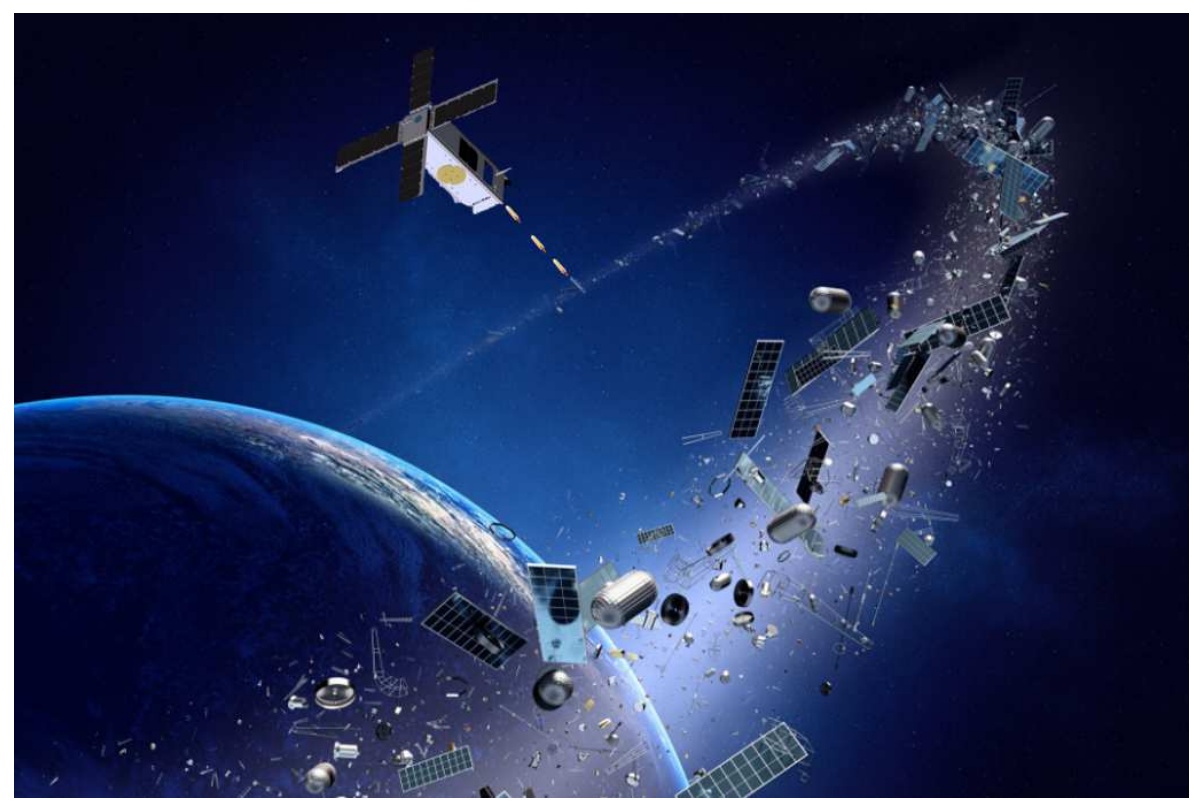

Fig. 2: Gun Shoot Concept for Deorbiting Debris

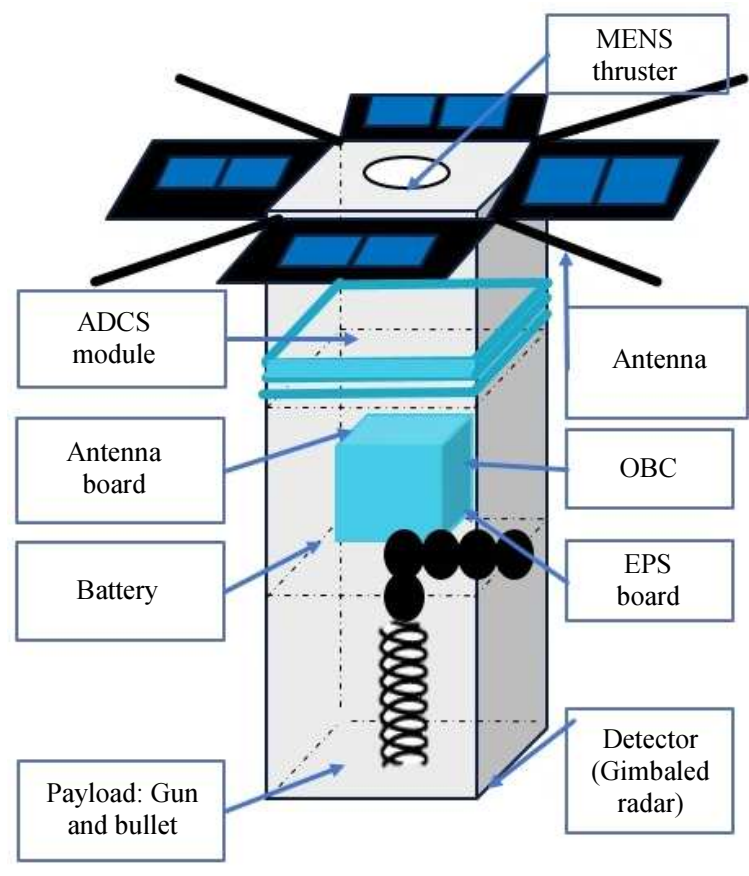

\begin{tabular}{|c|c|}
\hline \multicolumn{2}{|c|}{ Physical properties } \\
\hline Total mass & $3-5 \mathrm{~kg}$ \\
\hline Dimension $(\mathrm{mm})$ & $100 \times 100 \times 300$ \\
\hline
\end{tabular}

\begin{tabular}{|l|}
\hline Top $_{1}$ U: Thruster \\
\hline 1. MEMS thruster \\
\hline 2. Antenna \\
\hline 3. Solar panel \\
\hline 4. ADCS module \\
\hline Middle ${ }_{1}$ : BUS \\
\hline 1. OBC \\
\hline 2. EPS \\
\hline Bottom1U: Payload \\
\hline 1. Gun and bullet \\
\hline 2. Detector (TIRA) \\
\hline
\end{tabular}

Fig. 3: CubeSat Designing with Gun and Bullets Platform Configuration 

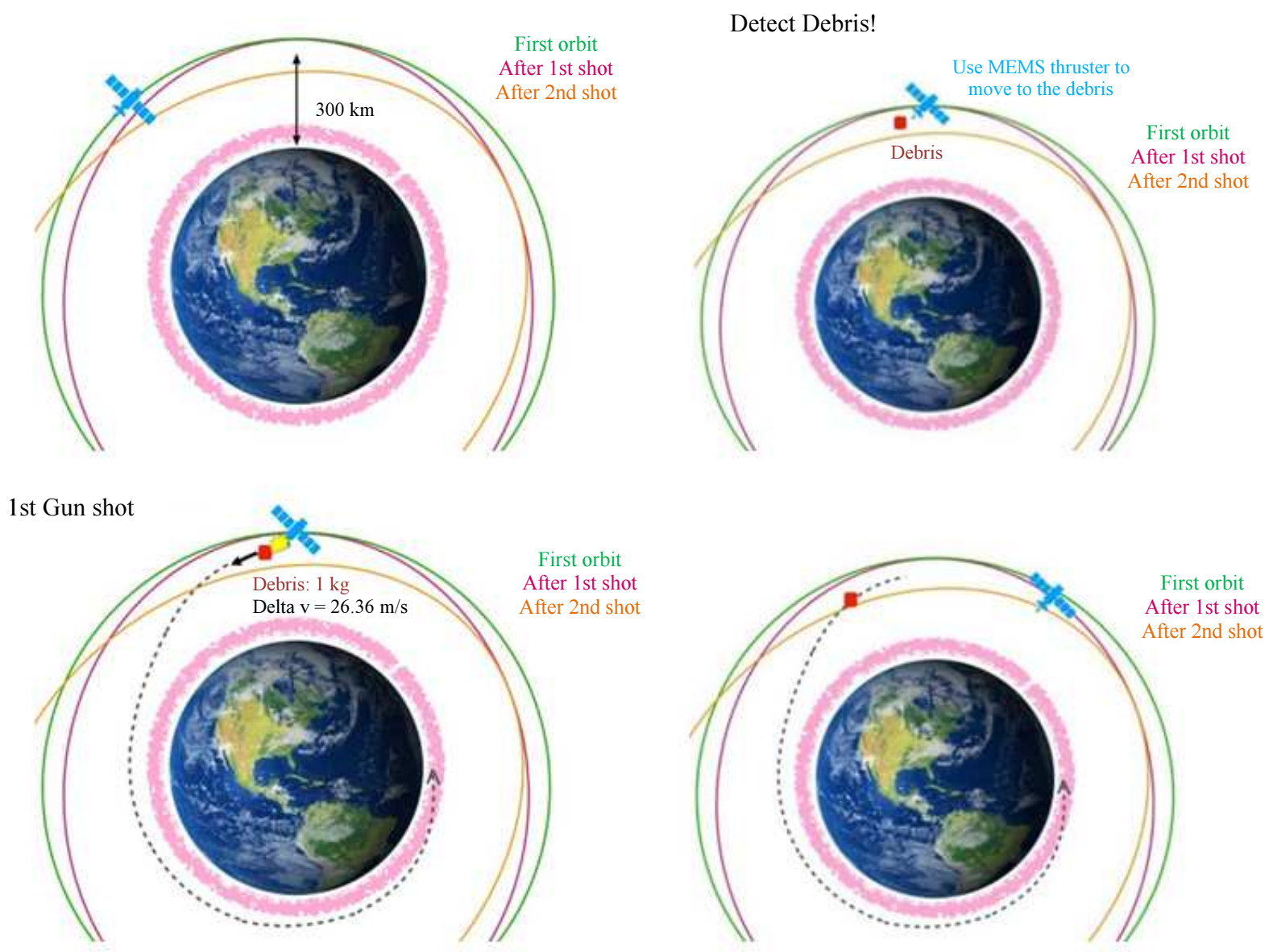

Fig. 4: Gun Shot Working Mechanism for Debris Removable

The Gun design has been driven by the bullets and CubeSat's weight, capacity. Those requirements led to exploit a Gun explosion to get the initial impulse for the bullet. As consequence, the Gun pressure become a sizing quantity, together with the bullets mass and emission velocities:

$$
v_{\text {ini-bullet }}=I_{\text {bullet }}=\int P \cdot A . d t
$$

The bullets mass plays a fundamental role in assuring a correct target of the debris. The single bullet mass is $0.1 \mathrm{Kg}$. The satellite going to change orbit by the reaction force of the Gun shoot. Then it can easily find out another debris for the next shoot.

\section{The Gunshot Working Mechanism}

In this mechanism, we have to detect debris with the help of a radar sensor and ground observation confirmation. Calibration of the data acquired by using sampling radars can be achieved by a number of techniques. These include radar range measurements of fragments of known size, shape and mass and the use of orbital calibration spheres. After calibration small satellite will approach to the detected debris for this MEMS thruster will work. This satellite will load five $0.1 \mathrm{~kg}$ bullet with strong thruster gunshot technology. Fig. 4 shows the working mechanism and debris removable process in details.

The delta $\mathrm{v}=26 \mathrm{~m} / \mathrm{s}$ for one shot and after the first Gunshot; the reaction force will help to change the orbit of the satellite to find out the second one space debris. Hysteresis damper passive attitude stabilization with the help of magnetic field after the shoot. After all, this mechanism helps to decrease the altitude of debris from $300-270 \mathrm{~km}$. The decay time shall be a few months to years. Fig. 5 depicts the sequence of the proposed gunshot working mechanism for debris deorbiting.

\section{System Block Diagram}

The power system being designed is the part of a much larger electrical system. Fig. 6 illustrate the system components involved in operating the satellite. The green block in the center represents the communications board, which is responsible for all the data collection and transmission, as well as any control systems management within the satellite. 


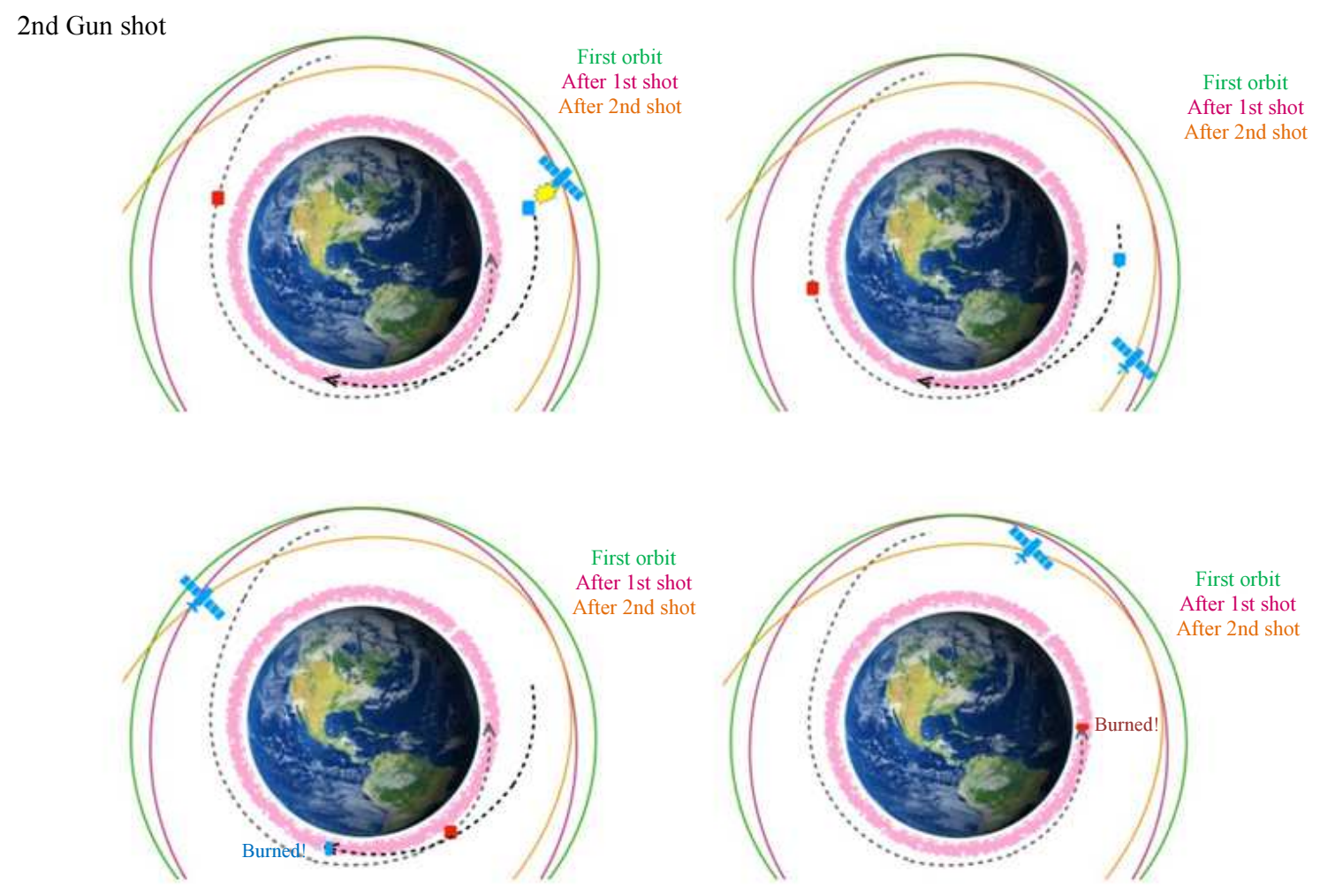

Fig. 5: Sequence of Gun Shot Working Mechanism for Debris Removable

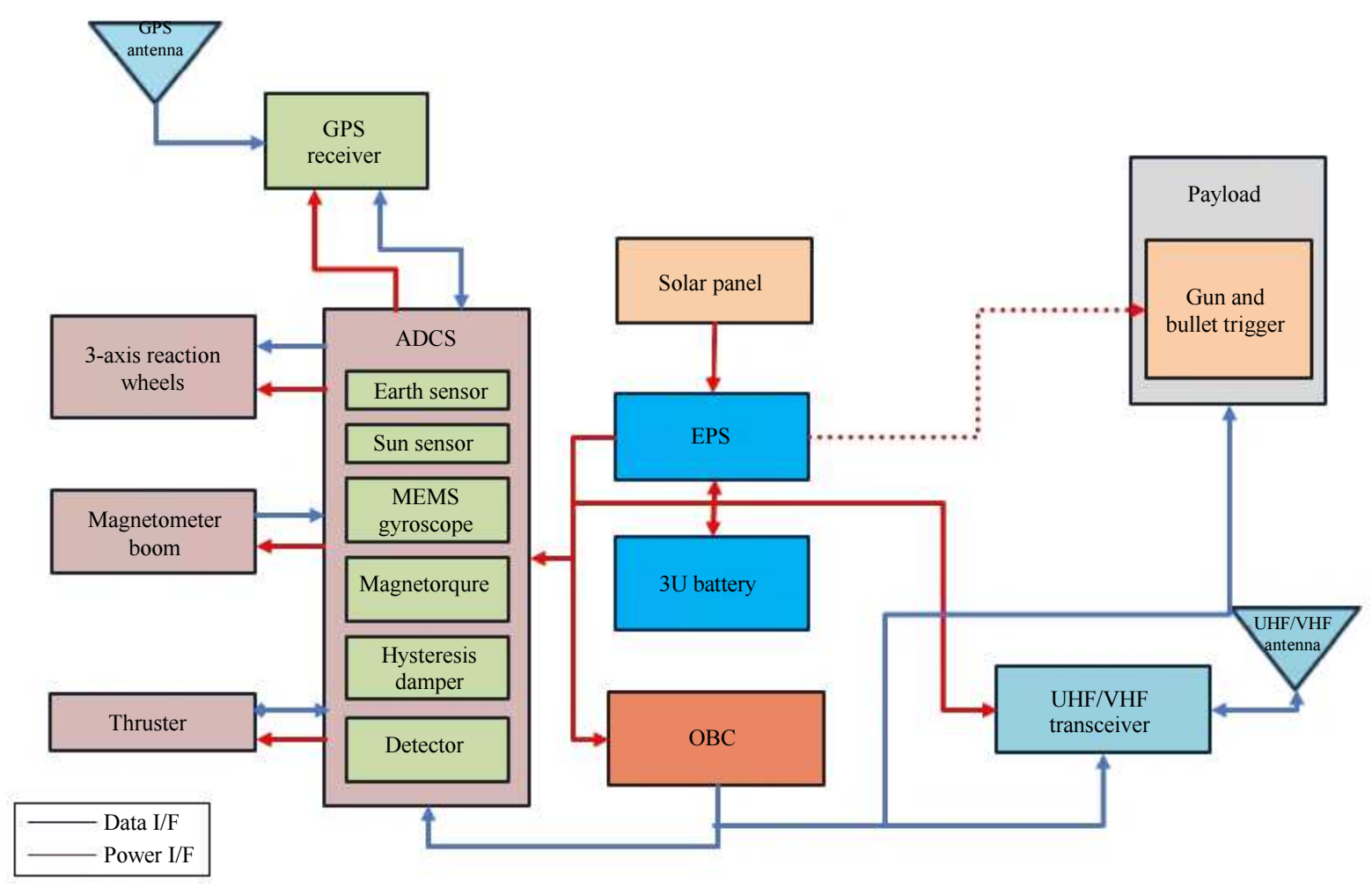

Fig. 6: System Block Diagram 
The power input comes from a system of 4 solar panels. One panel is attached to each long side of the CubeSat from so that while the satellite is rotating in space it will always be exposed to sunlight. The CubeSat Structural Subsystem is made of a lightweight material that provides adequate interfaces to each other subsystem to ensure safe passage through all phases of the mission. The ease of fabrication and assembly, light-weight and free space for the payload sensors, circuitry and batteries are the key features of the CubeSat structural subsystem design. The structural subsystem also has the ability to accommodate multiple payload sensors integrated in the subsystem in a simple manner.

\section{Conclusion}

This research paper allows elaborating on the direction and ideas for the future research concepts, as with the more technical oriented investigation on deorbiting space junks with indorsing results. The new theoretical concept and numerical simulations was conducted to investigate the feasibility of a Gunshot system to remove the space junk. The working mechanism and numerical calculation of a CubeSat with gunshot ability were developed conceptually in this study. Full-scale ground experiments will be performed to validate the numerical model and the test the development quality of the Gunshot under different approach easily. In addition, the small satellite with the gunshot technology model system could provide a better realistic working performance than the traditional debris removable models. This concept provides multiple space debris removal capacity and decreases the decay time of that debris effectively. The mechanism doesn't require large power and propulsion, can be applied to different material's junk and it will overcome the demerits of existing derbies removable techniques. So this concept is more effective in CubeSat and other small satellites to deorbiting the small sized LEO's space junks. A critical opportunity exists to mitigate and minimize the potential damage of satellites and ensure the sustainable development of the near-earth space environment; after instituting effective space debris removal mechanism.

\section{Acknowledgment}

The acknowledgement goes to all my professors, seniors and colleagues.

\section{Author's Contributions}

All authors equally contributed in this work.

\section{Ethics}

This article is orginal and unique and it is confirmed that all authers have read and approved the manuscript, there are no ethical issues in volved.

\section{References}

Ansdell, M., 2010. Active space debris removal: Needs, implications and recommendations for today's geopolitical environment. J. Public Int. Affairs, 21: 7-22.

Bastida, B. and H. Krag, 2011. Analyzing the criteria for a stable environment. Proceedings of the AAS/AIAA Astrodynamics Specialist Conference, (ASC' 11), Girdwood, Alaska.

Benvenuto, R. and R. Carta, 2013. Active debris removal system based on tethered-nets: Experimental results. Proceedings of the 9th Pegasus-AIAA Aerospace Student Conference, (ASC' 13), Politecnico di Milano, Italy.

Bonnal, C., J.M. Ruault and M.C. Desjean, 2013. Active debris removal: Recent progress and current trends. Acta Astronautica, 85: 51-60.

DOI: $10.1016 /$ j.actaastro.2012.11.009

COPUOS, 2007. Space debris mitigation guidelines of the scientific technical subcommittee of the committee on the peaceful uses of outer space.

David, W., 2007. Space debris. Phys. Today, 60: 35-40. DOI: $10.1063 / 1.2800252$

Gołębiowski, W., R. Michalczyk, M. Dyrek, U. Battista and K. Wormnes, 2016. Validated simulator for space debris removal with nets and other flexible tethers applications. Acta Astronautica, 129: 229-240. DOI: $10.1016 /$ j.actaastro.2016.08.037

Johnson, N.L., 2009. Statement before the house subcommittee on space and aeronautics. Committee on Science and Technology.

Kessler, D.J. and B.G. Cour-Palais, 1978. Collision frequency of artificial satellites: The creation of a debris belt. J. Geophys. Res., 83: 345-339. DOI: 10.1029/JA083iA06p02637

Liou, J.C., 2009. A sensitivity study of the effectiveness of active debris removal in LEO. Acta Astronautica, 64: 236-243. DOI: 10.1016/j.actaastro.2008.07.009

Mehrholz, D., L. Leushacke and W. Flury, 2002. Detecting, tracking and imaging space debris. ESA Bull., 109: 128-134.

Nielsen, P.D., K.T. Alfriend, M.J. Bloomfield, J.T. Emmert and Y. Guo et al., 2012. Continuing Kepler's quest: Assessing air force space command's astrodynamics standards. Tech. Rep., National Research Council, Washington, D.C. 
Nishida, S. and T. Yoshikawa, 2003. Space debris capture by a joint compliance controlled robot. Proceeding of the IEEE/ASME International Conference on Advanced Intelligent Mechatronics, Jul. 20-24, IEEE Xplore Press, Kobe, Japan, pp: 496-502. DOI: 10.1109/AIM.2003.1225145

NPDPO, 2007. Chinese anti-satellite test creates most severe orbital debris cloud in history. NASA Orbital Debris Quarterly News; NASA Orbital Debris Program Office, Houston, TX, USA.
NPDPO, 2009. Satellite collision leaves significant debris clouds. NASA Orbital Debris Quarterly News; NASA Orbital Debris Program Office, Houston, TX, USA.

Sannigrahi, A.K., 2017. Removal of active man-made orbital debris. Int. J. Sci. Res., 6: 827-832.

Wheeler, J., 2007. The current legal framework associated with space debris mitigation. Proc. IMechE, 221: 911-914.

DOI: $10.1243 / 09544100 J A E R O 168$ 